\title{
Fractal Flux Tubes of the Solar Magnetic Field
}

\author{
Alexander Ruzmaikin ${ }^{1}$, Dmitry Sokoloff ${ }^{2}$, Theodore Tarbell ${ }^{3}$ \\ ${ }^{1}$ IZMIRAN, Troitsk, Moscow Region 142092, USSR \\ ${ }^{2}$ Moscow State University, Moscow, USSR \\ ${ }^{3}$ Lockheed Palo Alto Research Labs, Palo Alto, CA 94304, USA
}

\begin{abstract}
The small-scale solar magnetic field exceeding a given threshold forms a fractal set. A dimension of this fractal is found from magnetograms with varying linear resolution. The dimension depends on the value of the threshold magnetic field (multifractality). A simple dynamo model explaining the origin of the fractal magnetic structure is considered. The dynamo produces a magnetic field in the form of flux tubes with a fractal distribution of magnetic field across the tube. The observed dimension gives a possibility of estimating a degree of structuredness of the solar velocity field.
\end{abstract}

\section{Introduction}

Analysis of many spectroscopic observations suggests that a great part of the solar magnetic field, except for a weak mean field, is concentrated in kilogauss flux tubes too small to be observed directly (they have diameters of order or less than 1000 $\mathrm{km}$ ), see a review by Stenflo (1989) and a recent paper by Tarbell et al. (1990b).

Dynamo theory predicts that a random flow of highly conducting fluid can lead to self-excitation of an initially weak magnetic field (Molchanov et al. 1983). However, the field grows highly inhomogeneously (intermittently): ropes and layers of intense field are prominent against a background of relatively slowly growing field. This was also demonstrated by numerical simulations (Meneguzzi et al. 1981). These ropes and layers form a network which randomly changes in space and time.

The solar magnetic field can be divided into a mean field, fluctuations of the mean field and a random magnetic field independently excited by random convective motions. In this paper we will be concerned with the interpretation of the random magnetic field, leaving aside the weaker and larger-scale mean field and its fluctuations which are important in the context of the solar cycle. Of course, the fluctuations of the mean field also may have a fractal structure. They probably tend to be vertical because the mean field emerges at the solar surface due to buoyancy. In a model approximation the fluctuating field can be considered as 
vertical and therefore be transported like a passive scalar which is stretched and subdivided by fine-scale turbulent motions into a fractal subset. At present it is difficult to distinguish these fluctuations from the random field excited independently. In principle it can be done by studying the solar cycle phase dependence and the correlation with the sunspots.

The fraction of the total volume occupied by the field structures in which the field strength exceeds a given level can serve as a quantitative measure of such intermittent field distribution. However, this part of the total volume at large magnetic Reynolds numbers can turn not to be a volume (even a small one). Due to the strong intermittency, the magnetic structures can occupy a manifold of a noninteger (fractal) dimension. Strictly speaking, at large but finite magnetic Reynolds numbers this manifold has the form of a volume. However in the solar conditions a characteristic scale for these ropes is too small (of the order of the electromagnetic skin-depth) to be observable so it is justified to consider this manifold as fractal. An adequate measure of the field distribution is the dimension of this manifold rather than the fraction of the total volume occupied by magnetic structures (which depends on the chosen resolution).

\section{The observed fractal dimension}

When some surface is observed at the linear resolution $\epsilon$, the fraction of the surface area occupied by the concentrations of magnetic field is given by

$$
S(\epsilon)=S_{\mathrm{f}} \epsilon^{2-d}
$$

where $d$ is known as the fractal dimension. It is clear that for $d=2$, i.e. when the concentrations occupy the whole surface, the area (1) does not depend on the resolution. In the other limiting case, when $d=0$ and the concentrations are actually points, we have $S(\epsilon) \propto \epsilon^{2}$, i.e. circles of radius $\epsilon$, which cover individual concentrations, do not overlap. Instead of $S$ one can use the number of pixels covering the fractal set, $N=S / \epsilon^{2}=S_{\mathrm{f}} \epsilon^{-d}$.

The value $S_{f}$ we may call "a fractal area". For a given solar box under observation $S_{\mathrm{f}}$ can be excluded by normalization. However it may appear that by observing the different boxes one will find different $S_{\mathrm{f}}$ 's. We guess that this value is proportional to the number of flux tubes filling the observational box. In principle the box can be filled up by one or two or more flux tubes having a complicated fractal form. The observational determination of $S_{\mathrm{f}}$ is thus meaningful.

Observations of solar magnetic fields at the scale of a single supergranulation cell with progressively higher resolution were first presented at the IAU Symposium No. 138 by Tarbell et al. (1990a) and later by Tarbell et al. (1990b). These first results, based on Kitt Peak magnetograms obtained at resolutions below 2.5 arc seconds and La Palma magnetograms at resolutions below 0.6 arc seconds, give only preliminary results for the fractal dimension $d$. The estimated value is about 1.6 over the range $0.6-10$ arc second considered, which indicates that the distribution of small-scale solar magnetic fields has fractal properties. An example of the 


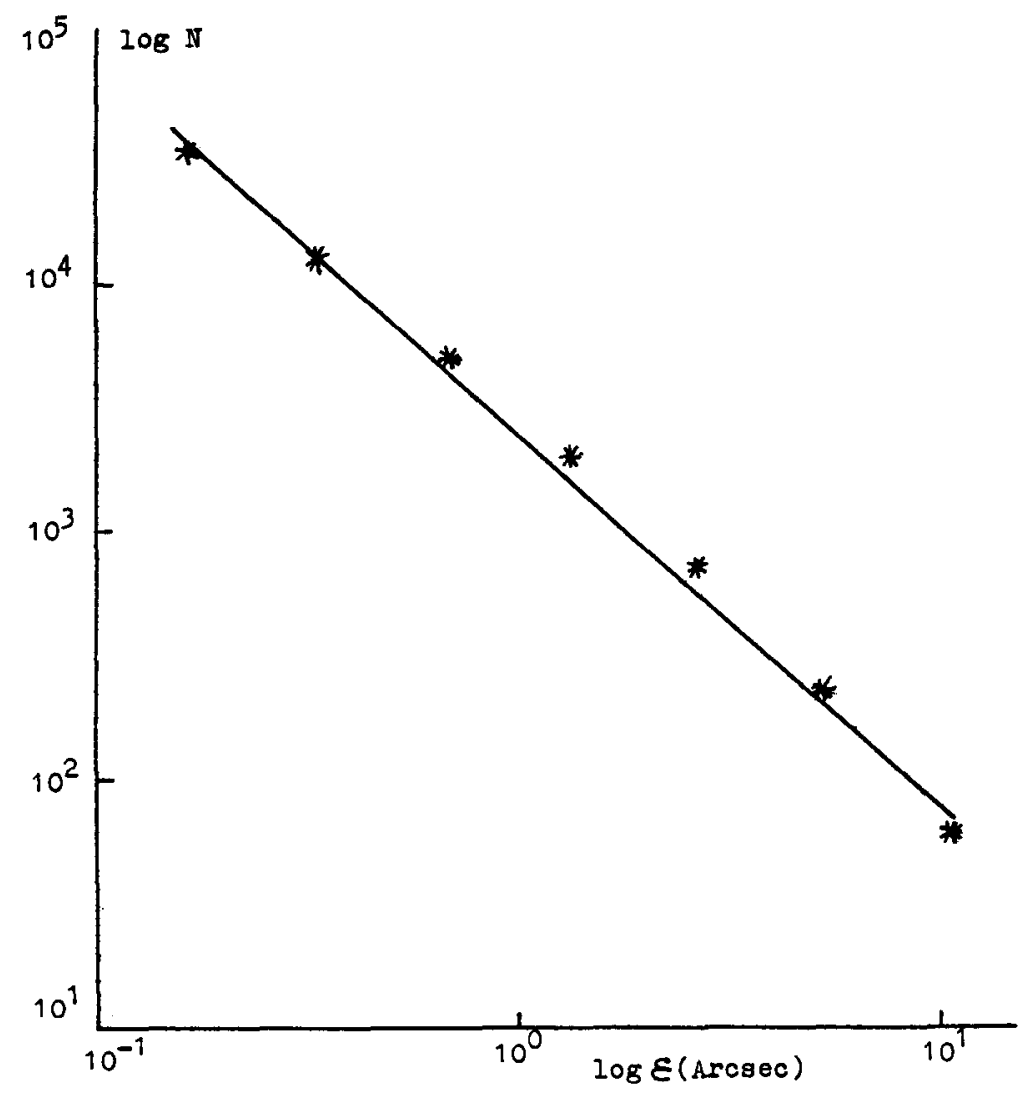

Fig. 1. The number of pixels covering the fractal set versus a pixel size. Threshold of the magnetograms used is $800 \mathrm{G}$. The resulting dimension is 1.49 (Tarbell et al., 1990b).

dependence of the number of pixels covering the area occupied by the fields whose strength exceeds a certain given value $H_{\text {th }}$ is given in Fig. 1. This dependence is linear when plotted in double logarithmic scale. The tangent of this line yields the desired dimension. Of course, the value of $d$ depends on the chosen threshold value of magnetic field strength. In that sense the magnetic field distribution can be described as a multifractal (the idea of multifractality is discussed by Frisch (1983) and recently by Zeldovich et al., 1990)

It can be expected that the fractal dimension decreases when the threshold field strength increases (Figs. 2 and 3) since the stronger the concentrations, the more rarely they are encountered.

Note that the fractal structure could hardly be revealed by Fourier power analysis, losing the important information about the phase correlations between different Fourier modes. Tarbell et al. (1990b) showed that the solar magnetic field spectrum is consistent with a power law $k^{-\sigma}$ where the exponent is close to $-3 / 2$ in the inertial range of the wavenumber space. This $3 / 2$ power law is exactly the 




Fig. 2. Dimension of magnetic fractal set versus the threshold magnetic field for the Kitt Peak magnetograms.

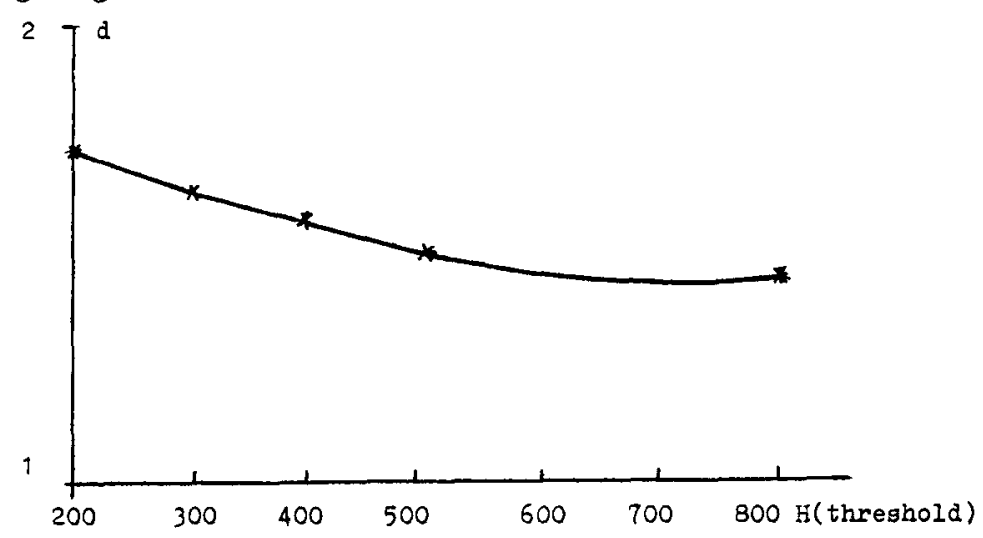

Fig. 3. $d$ versus $H$ (threshold) for the La Palma magnetograms.

known Kraichnan spectrum in the MHD turbulence of uncorrelated Alfvén waves. To catch the intermittency in this way it is necessary to find deviations from the $3 / 2$-law as accurately as possible.

\section{A dynamo model of the fractal distribution of magnetic field}

Twisting and folding of magnetic ropes form the basis of the dynamo in a random flow (see, e.g., Zeldovich et al., 1983). In the simplest case, the rope is stretched homogeneously to twice its size, which transformation is accompanied by a two-fold decrease of its cross-section because the magnetic flux is conserved. Consequent twisting and folding of the rope restores the cross-section and, thus, the magnetic field and flux are finally doubled. As noted by Finn and Ott (1988), the stretching 
can be inhomogeneous: some part of the rope can be stretched strongly (by the factor $\alpha^{-1}, \alpha<1 / 2$ ) while the remaining part is stretched only weakly (by the factor $(1-\alpha)^{-1}$, see Fig. 4). Obviously, in this case the field is stronger in that part of the rope which is stretched most strongly.
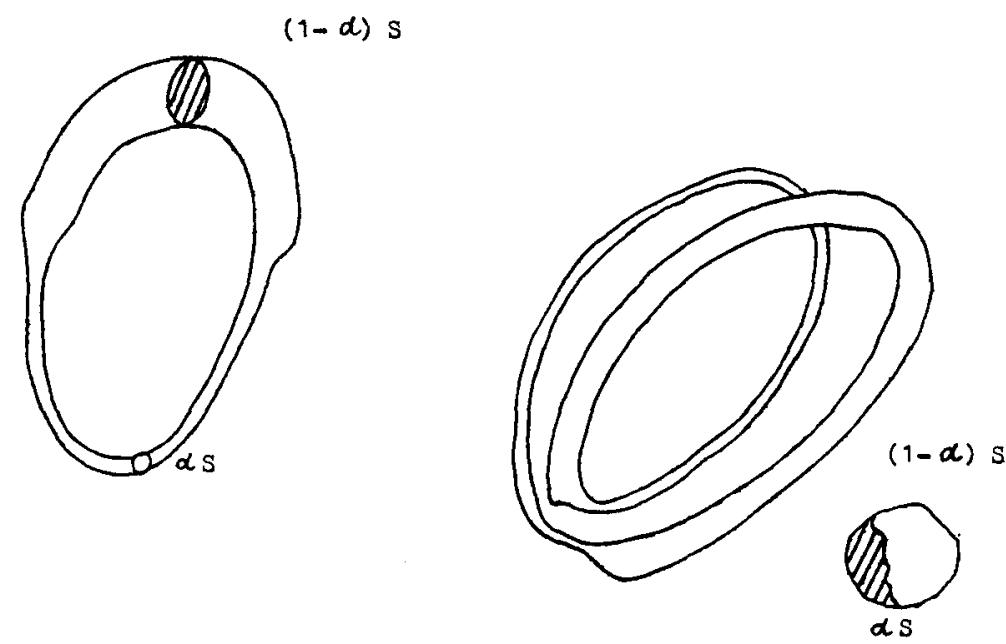

Fig. 4. The inhomogeneous stretching of magnetic rope results in a fractal distribution of the magnetic field over the cross-section of the rope.

Let us clarify the physical meaning of $\alpha$. This quantity characterizes a ratio of a turbulent cell size to an element of its structure. In that sense one can imagine the turbulent flow considered not as a structureless gaussian flow but as the structured random flow consisting of vortex lines with $\alpha$ characterizing a scale of these vortices.

After multiple repetitions of this transformation, the field concentrates within a small fraction of the initial loop cross-section. According to Finn and Ott (1988), the dimension of this part of the cross-section is given by

$$
d=1+\frac{\ln 2}{\ln [1 / \sqrt{\alpha(1-\alpha)}]} .
$$

The simplest case of the Zeldovich rope dynamo corresponds to $\alpha=1 / 2$ and $d$ $=2$. In a real flow the value of $\alpha$ varies from one loop to another whilst remaining between 0 and $1 / 2$. Thus, it can be expected that $d$ can vary from one position to another. In this sence, it would be correct to introduce an average dimension $d$ and consider deviations from this average value. However, as shown by equation (2), even strong variations in $\alpha$ only weakly affect the value of $d$. 


\section{Confrontation with observations and discussion}

The degree of inhomogeneity of the loop stretching can be determined from the observed value of $d$ (see Fig. 5).

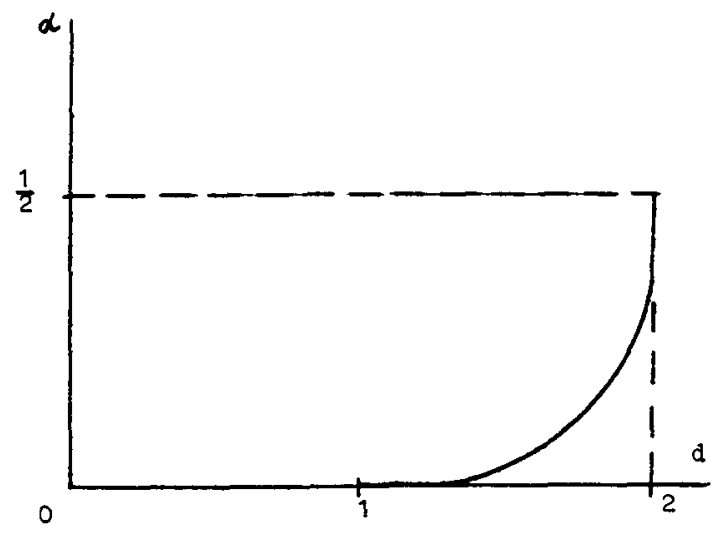

Fig. 5. Degree of structuredness of the flow versus the dimension of magnetic set.

In particular, for $d=1.2$ we have $\alpha \cong 10^{-3}$ and for $d=1.73$ we obtain $\alpha \cong 0.2$. Thus, the observed picture implies a highly inhomogeneous stretching of magnetic ropes, i.e. a high inhomogeneity of the velocity field.

While at this stage it is dangerous to make general conclusions it is clear that $\alpha$ is essentially smaller than $1 / 2$ corresponding to the simple Zeldovich model, which can be considered as a dynamo created by the structureless gaussian flow. The small value of $\alpha$ is an indication that the solar convective flow is highly structured in the observed cell. This deduction is well supported by observations of the solar velocity field. "Inside" the supergranule space under consideration there are, as it is well known, mesogranules and granules. In that sense the analysis of magnetic data may provide some evidence concerning the velocity field. The characteristic sizes of supergranule, mesogranule, and granule are of the order $3 \times 10^{4}, 8 \times 10^{3}$, and $10^{3} \mathrm{~km}$ correspondingly. Putting $\alpha=8 \times 10^{3} / 3 \times 10^{4}=0.27$ or $10^{3} / 3 \times 10^{4}=0.03$ one has $d=1.87$ or 1.5 . Thus the structuredness predicted from the observed dimension and the dynamo model is comparable with the structuredness of independently observed velocity fields.

The fractal distribution of the magnetic field over the cross-section of the tube causes a breakdown of the classical conception of the flux tube. We can introduce the concept of "a fractal flux tube" which has one aspect in common with the classical flux tube: there is no magnetic field perpendicular to the surface of the tube. Note however that in the dynamo model considered here it is assumed that the flux tube has a well defined surface. In the general case the boundary probably 
has a fractal form. That is evident from the study of the correlation properties of typical realizations of the magnetic field in a random flow (Galeeva et al., 1989).

\section{References}

Galeeva, R., Ruzmaikin, A.A., Sokoloff, D.D.: 1989, Magnitnaya Gidrodinamika No 3, 3 Finn, J., Ott, E.: 1988, Phys. Rev. Lett. 60, 760

Frisch, U.: 1983, in Chaotic Behaviour of Deterministic Systems, Les Houches Session, North Holland

Molchanov, S.A., Ruzmaikin, A.A., Sokoloff, D.D.: 1983, Geophys. Astrophys. Fluid Dyn. 30, 241

Meneguzzi, M., Frisch, U., Pouquet, A.: 1981, Phys. Rev. Lett. 47, 1060

Stenflo, J.O.: 1989, Astron. Astrophys. Rev. 1, 3

Tarbell, T., Ferguson, S., Frank, Z., Shine, R., Title, A., Topka, K.: 1990a, in Solar Photosphere: Structure, Convection and Magnetic Fields, ed. J.O. Stenflo, IAU Symp. No 138, Kluwer Academic Publishers, p. 147

Tarbell, T., Acton, D.S., Topka, K., Title, A., Schmidt, W., Scharmer, G.: 1990b, submitted to Phys. Fluids

Zeldovich, Ya.B., Ruzmaikin, A.A., Sokoloff, D.D.: 1983, Magnetic Fields in Astrophysics, Gordon and Breach

Zeldovich, Ya.B., Ruzmaikin, A.A., Sokoloff, D.D.: 1990, The Almighty Chance, World Sci. Pub., Singapore 